ARTIGO ORIGINAL ORIGINAL ARTICLE

Palavras-chave:

economia da saúde, neoplasias, políticas públicas de saúde

\section{Estratégias políticas que norteiam a incorporação de tecnologias: Avaliação de Tecnologias em Saúde em oncologia}

\author{
Policy strategies that drive the incorporation of technology: \\ Health Technology Assessment in oncology \\ Rodrigo Saar da Costa', Myrian Machado Fernandes', Orenzio Soler², Ligia Bahia³ \\ DOI: 10.21115/JBES.v9.n1.p30-8
}

\section{RESUMO}

Objetivo: Este trabalho tem como objetivo contextualizar os marcos regulatórios e as estratégias políticas no campo das Avaliações de Tecnologias em Saúde que norteiam as ações da rede de pesquisa clínica em oncologia e sua incorporação no Sistema Único de Saúde. Método: Trata-se de uma revisão do período entre 1988 e 2013, utilizando-se de descritores em Ciências da Saúde. Foram consultados, também, os bancos de dados do Departamento de Ciência e Tecnologia da Secretaria de Ciência, Tecnologia e Insumos Estratégicos do Ministério da Saúde, Agência Nacional de Vigilância Sanitária, Instituto Nacional de Câncer e Comissão Nacional de Incorporação de Tecnologias no Sistema Único de Saúde. Resultados: O Departamento de Ciência e Tecnologia é o responsável pela pesquisa em Avaliação de Tecnologias em Saúde no Brasil. Podem ser constatados avanços importantes nesse campo pelo governo brasileiro. Por fim, infere-se a necessidade de se aprimorarem as políticas e estratégias relacionadas ao processo de incorporação e de abandono de tecnologias, principalmente de tecnologias em saúde na área oncológica. Conclusão: As políticas científicas de controle do câncer devem estar cada vez mais alinhadas com as evidências científicas com objetivo de oferecer um sistema de saúde equânime e eficiente.

\begin{abstract}
Objective: This study aims to contextualize the regulatory frameworks and political strategies in the field of Health Technology Assessments that guide the actions of clinical research network in oncology and its incorporation into the National Health System. Method: This is a review of the period 1988-2013, using the Health Sciences Descriptors. Were consulted, too, the databases of the Department of Science and Technology of the Department of Science and Technology Strategic inputs from the Ministry of Health, the National Health Surveillance Agency, National Cancer Institute and the National Commission on Incorporation of Technologies. Results: Department of Science and Technology is responsible for research on Technology Assessment in Health in Brazil. It can be seen important advances in this field by the Brazilian government. Finally, it is inferred the need to improve the policies and strategies related to the process of development and abandonment technologies, especially health technologies in oncology. Conclusion: The scientific policies for cancer control will have to be increasingly aligned with scientific evidence in order to offer a fair and efficient health system.
\end{abstract}

\footnotetext{
Recebido em: 04/03/2016. Aprovado para publicação em: 20/01/2017.

1. Núcleo de Avaliação de Tecnologias em Saúde, Instituto Nacional de Câncer, Rio de Janeiro, RJ, Brasil.

2. Faculdade de Farmácia, Instituto de Ciências da Saúde, Universidade Federal do Pará (UFPA), Belém, PA, Brasil. 3. Instituto de Estudos em Saúde Coletiva, Universidade Federal do Rio de Janeiro (UFRJ), Rio de Janeiro, RJ, Brasil.

Conflitos de interesse: Nenhum dos autores recebe qualquer patrocínio da indústria ou participa de qualquer entidade de especialidade ou de pacientes que possa representar conflitos de interesse.

Autor correspondente: Rodrigo Saar da Costa. Instituto Nacional de Câncer José Alencar Gomes da Silva. Rua Marquês de Pombal 125, 70 andar - Centro, Rio de Janeiro, RJ, Brasil - 20230-240. E-mail: saarrc@gmail.com
} 


\section{Introdução}

As políticas científicas e os seus modelos e abrangência temática vêm sendo ampliadas nas últimas décadas, tendo por objetivo apoiar a produção de conhecimentos em saúde. Elas contribuem para a qualidade na atenção à saúde e para o desenvolvimento científico e tecnológico direcionado às necessidades de saúde das populações e, quando integradas às políticas de desenvolvimento econômico, produtivo e de inovação, apoiam o crescimento econômico do país (GadeIha, 2006; Guimarães, 2006; Banta \& Luce, 1993).

A Avaliação Econômica (AE) e a Avaliação de Tecnologia em Saúde (ATS) são reconhecidas como estratégicas para o desenvolvimento dos sistemas nacionais de saúde, assim como para a determinação explícita de benefícios em saúde, que implica a consolidação de instituições capazes de lidar com o delicado processo de integração entre ciência e política de saúde (Chinitz, 2004; Brasil, 2011). A responsabilidade técnica e política para todo o processo de ATS tem aumentado, estabelecendo-se como importante estratégia no processo de governança e gestão no campo da saúde pública e coletiva (Brasil, 2006b).

Nos últimos anos, o Brasil vem implementando distintas estratégias de sistematização do processo de definição de prioridades de pesquisa em saúde, com a finalidade de torná-la mais transparente, auxiliando os gestores públicos a tomarem decisões conscientes quanto à alocação adequada e produtiva dos fundos para pesquisa (Silva, 2003).

Os desfechos econômicos para decisões de priorizar a alocação de recursos vêm sendo mais bem reconhecidos no Brasil (Pichon-Riviere et al., 2010; Silva et al., 2012). Contudo, o uso e a aplicação de AE ou ATS ainda são limitados, pois há insuficiente conhecimento conceitual e técnico, dificuldades de acesso a estudos, falta de credibilidade em fontes de dados e na sua validade externa, bem como uma fragmentação institucional no setor da saúde (Novaes \& Elias, 2013; Banta \& Luce, 1993).

Compreende-se que mecanismos de priorização quanto ao financiamento de AE ou ATS são necessários, no sentido de assegurar as demandas emergentes e proporcionar maior harmonização de pesquisa em saúde, particularmente em combinação com os estudos de carga de doenças. Nos dias atuais, várias abordagens estão disponíveis para orientar na definição de prioridades de pesquisa em saúde, entretanto elas diferem quanto ao processo ideal em razão de diferentes contextos e práticas (Montorzi, 2010; Berg et al., 2004; Fleurence \& Torgerson, 2004; Kaplan \& Laing, 2004).

O envelhecimento da população brasileira, que projeta um crescimento exponencial de idosos, identifica um aumento expressivo na prevalência do câncer, o que demanda dos gestores do Sistema Único de Saúde (SUS) esforços para a oferta de assistência adequada aos pacientes (Goss et al., 2013; Brasil, 2006c). Esse cenário deixa claro a necessidade de maior investimento na prevenção de doenças e da modificação dos padrões de exposição aos fatores de risco para o câncer (Paim et al., 2010; Sestelo et al., 2013; Brasil, 2006a).

Este trabalho contextualiza, por meio de uma revisão, os marcos regulatórios e as estratégias políticas no campo das ATS que norteiam o processo de incorporação de tecnologias em oncologia para o SUS.

\section{Método}

As fontes utilizadas para a pesquisa de normas e políticas que regulamentam a incorporação de tecnologias em saúde e de assistência oncológica no país foram obtidas nas páginas eletrônicas do Ministério da Saúde (MS), da Agência Nacional de Vigilância Sanitária (Anvisa) e do Instituto Nacional de Câncer (Inca). O material foi coletado no segundo semestre de 2013, tendo como recorte temporal o período de 1988 a 2013, sendo selecionados e organizados os marcos regulatórios do processo de incorporação tecnológica em saúde e da política de saúde em oncologia.

Além disso, foi realizada uma análise das propostas demandadas de incorporação tecnológica no SUS para a Comissão de Incorporação de Tecnologias (Citec) do Ministério da Saúde e a Comissão Nacional de Incorporação de Tecnologias (Conitec) no SUS, no sítio do Ministério da Saúde (http://portalsaude.saude.gov.br/index.php/o-ministerio/ principal/secretarias/sctie/conitec), desde o ano da criação da Citec até o segundo semestre de 2013. Em adição, fez-se um levantamento das redes de pesquisa e desenvolvimento relacionadas ao câncer nas páginas eletrônicas do MS e do Inca, com o objetivo de identificar os mecanismos que o MS vem utilizando no sentido de concentrar esforços e financiar o crescimento tecnológico e científico.

É importante registrar que, desde 2002, o MS, por meio do Departamento de Ciência e Tecnologia (Decit), vem fomentado editais em ATS, em parceria principalmente com o Conselho Nacional de Desenvolvimento Científico e Tecnológico (CNPq). Os editais nacionais divulgados pelo MS são organizados por temas específicos e se propõem a fortalecer a ciência e a tecnologia em nível nacional, incentivando a livre concorrência. A consulta realizada foi feita no endereço eletrônico do Decit (http://pesquisasaude.saude.gov. $\mathrm{br} / \mathrm{bdgdecit/),com} \mathrm{o} \mathrm{intuito} \mathrm{de} \mathrm{analisar} \mathrm{os} \mathrm{projetos} \mathrm{de} \mathrm{ATS}$ em oncologia. A base de dados disponibiliza informações sobre projetos de pesquisas apoiados pelo Decit a partir de 2002 com a colaboração de instituições de apoio à pesquisa.

\section{Resultados e discussão}

\section{Contexto regulatório de ATS e de oncologia}

A busca pelo equilíbrio entre os desafios do SUS e o controle de gastos em saúde, sem redução do acesso e estagnação 
do desenvolvimento, resultou, a partir de meados da década de 1990, na implantação de importantes marcos regulatórios relacionados com o processo de avaliação e incorporação tecnológica em saúde no Brasil.

Como ponto de partida, dá-se destaque ao Projeto de Reforço à Reorganização do Sistema Único de Saúde (ReforSUS), em 1996; a criação do Decit, em 2000; a criação da Secretaria de Ciência, Tecnologia e Insumos Estratégicos (SCTIE) e do Conselho de Ciência, Tecnologia e Inovação em Saúde do Ministério da Saúde (CCTI), em 2003, sendo este responsável por conduzir as diretrizes e promover a avaliação tecnológica para a incorporação de novos produtos e processos pelos gestores, prestadores de saúde e de profissionais dos serviços no SUS.

Institui-se, em 1998, a Política Nacional de Medicamentos, como parte integrante da Política Nacional de Saúde (Portaria GM/MS no 3.916, de 30 de outubro de 1998), tendo como propósito precípuo garantir a necessária segurança, eficácia e qualidade dos medicamentos, a promoção do uso racional e o acesso da população àqueles considerados essenciais.

Ainda em 1998 é dado um importante passo na assistência oncológica por meio das Portarias no 3.535 e no 3.536 do Ministério da Saúde, ambas publicadas em 2 de setembro, tendo como objetivos: garantir atendimento integral aos pacientes com câncer; estabelecer uma rede hierarquizada dos centros que prestam assistência oncológica; atualizar os critérios mínimos para o cadastramento de Centros de Alta Complexidade em Oncologia (Cacon). As portarias determinavam, também, a implantação do Sistema de Autorização de Procedimentos de Alta Complexidade na área de oncologia (Apac/Onco), passando os novos procedimentos a serem baseados na neoplasia, e não mais nos medicamentos, para o tratamento oncológico.

A criação da Anvisa (Lei no 9.782, de 26 de janeiro de 1999) teve um importante papel no processo regulatório, possuindo como um de seus principais lócus de intervenção a regulação das tecnologias em saúde. A Anvisa atua no processo de incorporação tecnológica no sistema de saúde brasileiro, registro de produtos de interesse para a saúde e monitoramento de efeitos adversos, para fins de restrição de uso e retirada do mercado.

O Decreto no 4.766, de 26 de junho de 2003, instituiu a Câmara de Regulação do Mercado de Medicamentos (CMED), que tem por objetivo a adoção, implementação e coordenação de atividades relativas à regulação econômica do mercado de medicamentos por meio de mecanismos que estimulem a oferta de medicamentos e a competitividade do setor.

Por meio da Portaria GM/MS no 1.418, de 24 de julho de 2003, o Ministério da Saúde criou o Conselho de Ciência, Tecnologia e Inovação, tendo como principais atribuições: definir as bases da Política Nacional de Ciência, Tecnologia e Inovação em Saúde (PNCTIS); implementar e acompanhar os modelos de gestão para as ações de fomento científico e tecnológico no âmbito do MS; participar da elaboração, implementação e acompanhamento da Agenda Nacional de Prioridades de Pesquisa em Saúde, estabelecendo prioridades de pesquisa e sua disseminação, definindo diretrizes e promovendo a avaliação tecnológica com vistas à incorporação de novos produtos e processos pelos gestores no âmbito do SUS.

A Anvisa, por meio da Resolução da no 220, de 21 de setembro de 2004, define o regulamento técnico de funcionamento dos Serviços de Terapia Antineoplásica (STA), norma essa aplicável a todos os estabelecimentos públicos e privados do país que realizam atividades de terapia antineoplásica.

Em 2005, o MS implantou a PNCTIS, como parte integrante da Política Nacional de Saúde, formulada no âmbito do SUS.

O ano de 2005, no âmbito da Política de Combate ao Câncer, também foi marcado por importantes normativas que atualizaram e ampliaram as estratégias governamentais no SUS:

- Portaria GM/MS no 2.439, de 8 de dezembro de 2005, do MS, que instituiu a Política Nacional de Atenção Oncológica e possui como principais objetivos: desenvolver estratégias coerentes com a política nacional de promoção da saúde; organizar uma linha de cuidados que perpasse todos os níveis de atenção e de atendimento; constituir Redes Estaduais ou Regionais de Atenção Oncológica para acesso e atendimento integral; ampliar a cobertura (universalidade, equidade, integralidade, controle social e acesso); fomentar, coordenar e executar projetos estratégicos de incorporação tecnológica.

- Portaria GM/MS no 741, de 19 de dezembro de 2005, do MS, que define as Unidades de Assistência de Alta Complexidade em Oncologia, os Cacon, os Centros de Referência de Alta Complexidade em Oncologia e seus papéis na atenção à saúde.

Outrossim, a necessidade do MS em redefinir uma política de gestão de tecnologias em saúde e estabelecer o fluxo para sua incorporação no SUS mostrou-se necessária, tendo em vista o interesse de implementar, monitorar e difundir a ATS.

Nesse sentido, foi instituída a comissão para a elaboração da Política Nacional de Gestão de Tecnologias em Saúde (PNGTS) pela Portaria GM/MS n².510, de 19 de dezembro de 2005, coordenada pelo Decit. O produto gerado foi um documento contendo objetivos, princípios, diretrizes de atuação e responsabilidades institucionais relativas à gestão de tecnologias no SUS.

A Anvisa, por meio das resoluções RDC no 185 e RE n³.385, ambas de 13 de outubro de 2006, passou a exigir informações econômicas para produtos, considerando a necessidade de diminuir a assimetria de informações referentes a produtos para a saúde. Essa medida constitui fundamental instrumen- 
to para o trabalho dos envolvidos em atividades de gestão em saúde e para maior esclarecimento da população usuária desses produtos, além de definir a lista de produtos sujeitos à apresentação dessas informações econômicas.

O MS, por meio da Portaria GM/MS no 3.323, de 27 de dezembro de 2006, instituiu a Citec, tendo como missão deliberar sobre solicitações de incorporação de tecnologias, análise de tecnologias em uso, revisão e mudanças de protocolos em consonância com as necessidades sociais em saúde e de gestão do SUS, bem como da Saúde Suplementar, passando a ser vinculada à SCTIE do MS por meio da Portaria GM/MS n².587, de 30 de outubro de 2008.

Em adição, entre 2006 e 2008, a Citec foi coordenada pela Secretaria de Atenção à Saúde (SAS) e em outubro de 2008 foi transferida para a SCTIE, quando foram redefinidas suas competências e o fluxo para incorporação de tecnologias em saúde no SUS. Estabeleceram-se, como de sua competência, a recomendação de incorporação ou retirada de produtos de saúde da lista de procedimentos do SUS e do rol de procedimentos da Agência Nacional de Saúde Suplementar (ANS), a proposição da revisão de diretrizes clínicas e protocolos terapêuticos e assistenciais de interesse para o sistema público de saúde e a solicitação da realização de estudos de ATS para subsidiar as atividades de análise e recomendação.

Nos anos de 2008 e 2009 foram lançados pelo MS manuais e diretrizes metodológicas com o objetivo de facilitar o acesso a informações sobre incorporações tecnológicas em saúde e a disseminação delas. Identificou-se a necessidade de se institucionalizar uma política com diretrizes nacionais para a implantação da avaliação, incorporação e gestão de tecnologias em saúde. Nessa direção, a Portaria GM/MS no 2.690, de 5 de novembro de 2009, institui a PNGTS, tendo como objetivo maximizar os benefícios à saúde, assegurando o acesso da população a tecnologias efetivas e seguras, em condições de equidade.

O papel do Decit consistia na produção e adoção de providências para o desenvolvimento de estudos com o objetivo de subsidiar as decisões na Citec. Em 2009, essa função foi formalmente incluída na estrutura regimental do MS, que tem a atribuição de coordenar a formulação e a implementação de políticas, programas e ações de avaliação de tecnologias no SUS. Desde então novas alterações ocorreram na estrutura da SCTIE, e em 2011 a Citec passou a ser denominada Conitec, ficando sob a responsabilidade de um novo departamento da SCTIE: o Departamento de Gestão e Incorporação de Tecnologias em Saúde - DGTIS (Novaes \& Elias, 2013).

A Lei no 12.401, de 28 de abril de 2011, foi um importante passo no processo regulatório de incorporação tecnológica em saúde, alterando a Lei no 8.080, de 19 de setembro de 1990, e dispondo sobre a assistência terapêutica e a incorporação de tecnologia em saúde no âmbito do SUS. Ela esta- belece os limites para o financiamento da saúde brasileira e regulamenta a Conitec, tendo como atribuição a incorporação, exclusão ou alteração de novos medicamentos, produtos e procedimentos, bem como a constituição ou alteração de protocolos clínicos ou de diretriz terapêutica.

Nesse contexto, a Política Nacional de Câncer - com o objetivo de adequação aos novos contextos político, social, epidemiológico e de financiamento da saúde - foi revisada e revogada pela Portaria GM/MS no 874, de 16 de maio de 2013, instituindo a Política Nacional para a Prevenção e Controle do Câncer na Rede de Atenção à Saúde das pessoas com doença crônicas no âmbito do SUS. Essa política reconhece o câncer como doença prevenível e a necessidade de oferta de cuidado integral ao paciente.

A Política Nacional para a Prevenção e Controle do Câncer estabelece que a organização das redes deve ocorrer de forma regionalizada e descentralizada, respeitando critérios de acesso, escala e escopo, além da necessidade de formação de profissionais e promoção da educação permanente. Determina ainda que a incorporação e o uso de tecnologias devem ser resultado de recomendações formuladas por órgãos governamentais, a partir de processos de AE e de ATS. Sua importância também está associada à definição da responsabilização para todas as esferas de gestão.

Ao Ministério da Saúde cabe definir normas gerais para a organização de linhas de cuidado, elaborar protocolos clínicos e diretrizes terapêuticas, fazer avaliação de tecnologias em saúde, efetuar a habilitação dos estabelecimentos e estabelecer diretrizes e recomendações para a prevenção e o controle do câncer.

Em convergência com a Lei no 12.401, de 28 de abril de 2011, as diretrizes relacionadas à ciência e à tecnologia no âmbito da Política Nacional para a Prevenção e Controle do Câncer prevê o estabelecimento de métodos e mecanismos para análise de viabilidade econômico-sanitária de empreendimentos públicos no Complexo Industrial da Saúde, direcionados para a prevenção e o controle do câncer, a implementação da rede de pesquisa para a prevenção e o controle do câncer, visando aumentar a produção de conhecimento nacional relacionada a essa área, bem como a implementação de práticas de elaboração de parecer técnico-científico, ATS e AE para subsidiar a tomada de decisão no processo de incorporação de novas tecnologias no SUS.

\section{Redes de pesquisa e desenvolvimento nacional}

A constituição de Redes de Pesquisa para a realização de estudos estratégicos foi uma das recomendações da PNGTS. O Ministério da Saúde vem fomentando a criação de redes técnico-científico-políticas nos últimos anos, com o objetivo de concentrar esforços no crescimento e financiamento tecnológico e científico. No contexto da oncologia, foram identificadas as seguintes redes: 


\section{- Rede Brasileira de Avaliação de Tecnologias em} Saúde (Rebrats): instituída em 2011, constitui-se por 63 instituições-membro, incluindo instituições de ensino e pesquisa e instituições gestoras, e pelos Núcleos de Avaliação de Tecnologias em Saúde em Hospitais de Ensino (NATS). Os NATS buscam introduzir a cultura de ATS em hospitais, por meio da utilização de evidências disponíveis para auxiliar o gestor hospitalar a tomar decisões quanto à inclusão de novas tecnologias, à avaliação de tecnologias difundidas e ao seu uso racional. A Rebrats tem como objetivo produzir e disseminar estudos e pesquisas prioritárias no campo de ATS, padronizar metodologias, validar e atestar a qualidade dos estudos, promover capacitação profissional na área, e estabelecer mecanismos para monitoramento de tecnologias novas e emergentes (Brasil, 2013a).

\section{- Rede Brasileira de Pesquisa sobre o Câncer} (RBPC): instituída em 2008, é um esforço conjunto do Ministério da Saúde e do Ministério da Ciência e Tecnologia para reunir as informações de pesquisas básicas e clínicas sobre o câncer no país, permitindo avanços no conhecimento e no fornecimento de subsídios para a tomada de decisões quanto às políticas de saúde. Também tem como objetivo agregar pessoas e formar novos recursos humanos na área de bioinformática para acompanhar e analisar os resultados de sequenciamentos futuros dos tumores.

- Institutos Nacionais de Ciência e Tecnologia (INCT): o programa INCT, instituído em 2008, foi planejado e implementado visando articular e mobilizar pesquisadores de todo o país, impulsionar a pesquisa básica e fundamental, tornando-a competitiva internacionalmente, estimular a pesquisa científica e tecnológica de ponta, promover a inovação e o espírito empreendedor, estabelecendo programas que contribuam para a melhoria do ensino de ciências e a difusão do conhecimento científico para a sociedade (Brasil, 2013b). Adicionalmente, buscou-se uma melhor distribuição nacional dos recursos para a pesquisa e a qualificação do país em áreas prioritárias para o seu desenvolvimento regional e nacional. No contexto da ATS e da oncologia, foram identificados quatro INCT:

- Instituto Nacional de Ciência e Tecnologia de Avaliação em Tecnologias em Saúde (INCT-IATS);

- Instituto Nacional de Ciência e Tecnologia para Controle do Câncer (INCT-PCC);

- Instituto Nacional de Ciência e Tecnologia das Doenças do Papilomavírus (INCT-HPV);

- Instituto Nacional de Ciência e Tecnologia em Oncogenômica (INCiTO).

- Rede Nacional de Desenvolvimento e Inovação de Fármacos Anticâncer (Redefac): a SCTIE do MS, considerando a necessidade de ações de pesquisa para a implementação de tecnologias terapêuticas inovadoras e que ampliem o acesso da população brasileira a medicamentos antineoplásicos, resolve instituir a Redefac, por meio da Portaria GM/ MS no 30, de 25 de setembro de 2012, com o objetivo de promover o desenvolvimento científico e tecnológico de fármacos, medicamentos e produtos para diagnóstico em oncologia, de forma a contribuir com a implementação de políticas públicas de controle do câncer.

\section{- Rede Nacional de Pesquisa Clínica em Câncer}

(RNPCC): instituída por meio da Portaria GM/ MS no 192, de 31 de janeiro de 2014, é composta por instituições de ciência, tecnologia, inovação e produção em saúde, pública e privada, com o objetivo de desenvolver atividades colaborativas na área de pesquisa em câncer; além de articular instituições de pesquisa clínica no âmbito do SUS, visando à realização de ensaios clínicos, à qualificação profissional e à implantação de protocolos clínicos e diretrizes terapêuticas voltados à melhoria da qualidade da atenção oncológica.

\section{Parcerias para o Desenvolvimento Produtivo (PDP)}

Outra estratégia importante foi regulamentada pela Portaria GM/MS MS/GM no 837, de 18 de abril de 2012, que define as diretrizes e os critérios para estabelecimento das Parcerias para o Desenvolvimento Produtivo (PDP), que trata de parcerias realizadas entre instituições públicas e entidades privadas, com vista ao acesso a tecnologias prioritárias, à redução da vulnerabilidade do SUS em longo prazo e à racionalização e redução de preços de produtos estratégicos para a saúde. Há o comprometimento de internalizar e desenvolver novas tecnologias estratégicas e de valor agregado elevado.

Outrossim, o marco regulatório das PDP está entre as medidas tomadas pelo MS para fortalecer a indústria nacional, tornando os produtores públicos e privados nacionais competitivos e capacitados a enfrentar a concorrência global. Até dezembro de 2012, havia 88 PDP articuladas pelo MS, compreendendo 77 produtos; sendo: 64 medicamentos, 7 vacinas, 4 produtos para saúde e 4 produtos de pesquisa e desenvolvimento.

Em 2013, foram assinadas 26 novas parcerias entre laboratórios públicos e privados para o desenvolvimento, incluindo biofármacos de última geração para o tratamento de câncer, artrite reumatoide e diabetes, e para ser usados como cicatrizante cirúrgico, hormônio de crescimento, vacina alergênica e contra o papilomavírus humano (HPV). O alvo são os produtos essenciais dos programas de saúde, como saúde da mulher, saúde mental e doenças crônicas, além de medicamentos que tiveram sua produção descontinuada pela indústria farmacêutica, com o caso da L-asparaginase. Está contemplada nessas novas parcerias a produção dos medicamentos adalimumabe, para artrite reumatoide, e trastuzumabe, incorporado recentemente para o tratamento de mulheres com câncer de mama. 
Outras cinco PDP já estão estabelecidas para o tratamento de leucemias/linfomas como a L-asparaginase e o rituximabe. Para os cânceres colorretal e de cabeça/pescoço, há o medicamento cetuximabe. Para os cânceres colorretal, renal, de ovário, pulmão e mama, há o bevacizumabe e o fator de crescimento hematopoiético filgrastim, empregado em uma série protocolos oncológicos (Quadro 1). Acredita-se que, a partir do desenvolvimento de linhas de produção desses biofármacos, o avanço tecnológico com o emprego de ferramentas semelhantes irá facilitar a incorporação de outros medicamentos ainda com o uso limitado pelo custo, assim como o desenvolvimento de novos medicamentos para o tratamento de câncer no país.

Para ser ter melhor compreensão sobre o impacto dessas estratégias, basta atentar que em 2013 o MS disponibilizou um orçamento de R\$ 35 bilhões para aquisição de medicamentos, e cerca de $60 \%$ das compras corresponde a biológicos. Os Medicamentos do Componente de Alto Custo têm consumido $43 \%$ dos recursos do MS, apesar de representarem somente $5 \%$ da quantidade adquirida. Assim, a partir do estabelecimento das PDP, o governo realizou uma série de arranjos para viabilizar a infraestrutura, a regulamentação e o aparato jurídico necessários para a produção nacional desses medicamentos.

Entre as ações propostas pela PDP estão parcerias entre laboratórios privados, nacionais ou internacionais, que detêm as patentes do medicamento de referência, para transferir a tecnologia para laboratórios públicos até que esses ganhem independência para produzir o medicamento. Em troca, o governo garante a compra do medicamento produzido internamente durante todo o período de transferência de tecnologia, estipulado em, no máximo, cinco anos.
Na linha do tempo, é importante registrar o Programa para o Desenvolvimento do Complexo Industrial da Saúde (Procis), regulamentado pela Portaria GM/MS no 506, de 21 de março de 2012, que, entre outras medidas de estímulo à produção pública, aumenta em cinco vezes os investimentos em 18 laboratórios públicos nacionais. Entre 2000 e 2011, o investimento total do governo nesse setor foi de $R \$ 512$ milhões. Em 2012, foram investidos R\$ 270 milhões na infraestrutura e qualificação de mão de obra desses laboratórios, e entre os anos de 2013 e 2016 o investimento foi da ordem de R\$ 2 bilhões, sendo R\$ 1 bilhão do governo federal e R\$ 1 bilhão de contrapartidas estaduais. Isso representa cinco vezes mais do que foi investido nos últimos 12 anos.

Outra estratégia foi a Lei no 12.349, 15 de dezembro de 2010, que estabelece margem de preferência de até 25\% nas licitações para empresas que produzam no Brasil. No âmbito do registro de medicamentos, a Anvisa e o Instituto Nacional de Propriedade Intelectual (Inpi) passam a cumprir, a partir de 2010, prazos mais curtos para registro de medicamentos prioritários ao SUS. Também foram criadas linhas de crédito para empresas que investem em biossimilares e equipamentos - um total de R\$ 7 bilhões, financiados pelo Banco Nacional de Desenvolvimento Econômico e Social (BNDES), Financiadora de Estudos e Projetos (Finep) e Ministério da Saúde.

A redução de gastos estimada pelo MS, proporcionada pelas parcerias para produção de medicamentos, insumos e equipamentos, é de $R \$ 550$ milhões por ano em compras públicas. Esse valor, somado à redução de custos gerada por inovação tecnológica e melhor gestão de recursos em vacinas, negociações e centralização de compras, pode levar a uma economia geral de R\$ 1,8 bilhão por ano no orçamento

Quadro 1. Parcerias para o Desenvolvimento Produtivo para biológicos no tratamento de câncer

\begin{tabular}{llll}
\hline $\begin{array}{l}\text { INDICAÇÃO* } \\
\text { TTIPO DE CÂNCER }\end{array}$ & PRODUTO & PARCEIRO PÚBLICO & PARCEIRO PRIVADO \\
\hline $\begin{array}{l}\text { Mama } \\
\text { Gástrico }\end{array}$ & Trastuzumabe & $\begin{array}{l}\text { Bahiafarma } \\
\text { Biomanguinhos } \\
\text { IVB** }\end{array}$ & $\begin{array}{l}\text { Libbs, Mabxience } \\
\text { Orygen, Alteogen } \\
\text { Bionovis }\end{array}$ \\
\hline $\begin{array}{l}\text { Colorretal } \\
\text { Cabeça e pescoço }\end{array}$ & Cetuximabe & $\begin{array}{l}\text { IVB, Biomanguinhos } \\
\text { Butantan }\end{array}$ & Bionovis \\
\hline $\begin{array}{l}\text { Leucemia } \\
\text { Fator de crescimento Mabxience }\end{array}$ & Fiocruz & NTharma \\
hematopoiético & L-asparaginase & Biotec \\
\hline $\begin{array}{l}\text { Linfoma } \\
\text { Leucemia }\end{array}$ & Filgrastim & Biomanguinhos & Eurofarma \\
\hline $\begin{array}{l}\text { Ovário, colorretal } \\
\text { pulmão, mama } \\
\text { renal }\end{array}$ & Rituximabe & IVB**, Biomanguinhos & Bionovis \\
Butantan & Libbs, Mabxience \\
\hline
\end{tabular}

* Indicações aprovadas pela Agência Nacional de Vigilância Sanitária. ** Instituto Vital Brasil. 
do Ministério da Saúde. Por meio dessas iniciativas, o Brasil agora faz parte de um seleto grupo de países que produz medicamento biotecnológico para câncer e outras doenças crônicas. A produção nacional diminui o risco de o Brasil ser surpreendido pela suspensão da produção de um medicamento por um laboratório privado internacional e incentiva uma cultura de independência tecnológica.

\section{Financiamento de pesquisa em Avaliação Econômica}

A PNGTS (Portaria GM/MS n 2.690, de 5 de novembro de 2009) propõe a promoção de parcerias com instituições de fomento, de modo a financiar as necessidades de pesquisa e desenvolvimento identificadas nas diversas etapas de gestão de tecnologias. Desde 2005, o Decit vem fomentando editais em ATS, em parceria com o CNPq, que totalizaram, entre 2005 e 2013, R\$ 18 milhões e 150 projetos aprovados. A participação da área oncológica está apresentada no Quadro 2.

Os recursos para o financiamento são provenientes do Fundo de Cooperação Bilateral do Ministério da Ciência e Tecnologia, contando com o apoio técnico-administrativo do CNPq e da Finep. A cooperação técnica entre os Ministérios da Saúde e da Ciência e Tecnologia incentiva e induz pesquisas em áreas prioritárias para o SUS.

O Decit tem realizado modalidades de fomento nacional e fomento descentralizado à pesquisa, tendo como objetivo aperfeiçoar a gestão e os serviços oferecidos pelo SUS à população. A modalidade de fomento nacional provê oportunidade para pesquisadores de todo o país se dedicarem a temas considerados prioritários para a saúde pública. Sua realização acontece por meio do lançamento de editais, em âmbito nacional, com o apoio técnico-administrativo do CNPq e da Finep.

A principal modalidade de fomento é a nacional, responsável por financiar $77 \%$ do total de projetos analisados. Aproximadamente $73 \%$ (R\$ $1.750 .240,86$ ) de todo o recurso investido nos projetos foram provenientes do Decit. Trinta por cento dos estudos/projetos são de análises econômicas, principalmente de custo-efetividade ou impacto orçamentário. Esses estudos (Quadro 2) tiveram como assunto principalmente o câncer de mama (sete estudos), seguidos de câncer do colo do útero/HPV (cinco estudos), leucemias/linfoma (quatro estudos) e câncer de próstata (três estudos).

A modalidade fomento descentralizado é feita por meio do Programa Pesquisa para o SUS: Gestão Compartilhada em Saúde (PPSUS), que visa à redução das desigualdades regionais na produção do conhecimento científico, fortalecendo a capacidade de gestão da política científica e tecnológica em saúde em todos os estados da federação. Desse modo, o PPSUS exerce papel importante na consolidação de uma cultura científica nacional, facilitando o estudo do perfil epidemiológico de populações locais.
É importante mencionar que, mesmo com o incentivo governamental da modalidade de fomento descentralizado, é observada uma concentração de projetos de ATS em oncologia na região Sudeste, em torno de $76 \%$, sendo $13 \mathrm{em}$ São Paulo e 8 no Rio de Janeiro, entre os anos de 2005 a 2013.

O investimento total por meio dos editais do Decit, para a área de ATS e AE em oncologia, foi de R\$ 2.391.903,49, equivalente a $12 \%$ do total de recursos investidos durante o período analisado. Registra-se que, do total de 10 editais, 30 proje-

Quadro 2. Projetos em ATS e Economia da Saúde em Oncologia financiados por meio de editais de pesquisa do Decit/MS entre o período de 2005 e 2013

\begin{tabular}{|c|c|}
\hline TEMAS AVALIADOS & TOTAL \\
\hline Tipos de tecnologia avaliada & 30 \\
\hline Procedimento/processo & 17 \\
\hline Produto & 5 \\
\hline Medicamento & 8 \\
\hline Tipos de estudos produzidos & 30 \\
\hline Avaliação Econômica & 9 \\
\hline $\begin{array}{l}\text { Avaliação de Programa } \\
\text { (diagnóstico/rastreamento) }\end{array}$ & 7 \\
\hline Avaliação de qualidade de vida & 5 \\
\hline $\begin{array}{l}\text { Pesquisa bibliográfica/estudo } \\
\text { de eficácia e segurança }\end{array}$ & 7 \\
\hline Outros* & 2 \\
\hline Tipos de câncer** & 25 \\
\hline Próstata & 3 \\
\hline Mama & 7 \\
\hline Colorretal & 1 \\
\hline Pulmão & 2 \\
\hline Leucemia/linfoma & 4 \\
\hline Colo uterino & 5 \\
\hline Tireoide & 1 \\
\hline Pele (melanoma) & 1 \\
\hline Renal & 1 \\
\hline Financiamento & 30 \\
\hline Região Sudeste & 21 \\
\hline Demais regiões & 9 \\
\hline Financiamento & 30 \\
\hline Fomento nacional & 23 \\
\hline Fomento descentralizado - PPSUS & 4 \\
\hline Contratação direta & 3 \\
\hline Origem dos recursos & $\mathrm{R} \$ 2.391 .903,49$ \\
\hline Recursos parceiros (principalmente CNPq) & $\mathrm{R} \$ 641.662,63$ (27\%) \\
\hline Recurso Decit & $\mathrm{R} \$ 1.750 .240,49(73 \%)$ \\
\hline
\end{tabular}

* Dois projetos de avaliação de desempenho e de genética. ** Os tipos de cânceres foram organizados segundo ordem de incidência da doença, tendo o câncer de próstata maior incidência. 
tos foram financiados tendo como tema principal a ATS e AE para o câncer.

Entre 2006 e 2011, foram totalizadas 20 demandas em oncologia para a Citec (Quadro 3). Dessas, nove solicitações foram aprovadas, sendo $70 \%$ dos demandantes do setor privado, em sua maioria da indústria farmacêutica. Em adição, a Citec recomendou a incorporação de quatro novos medicamentos para o tratamento de cinco tipos de câncer: dasatinibe, nilotinibe e imatinibe, para leucemia mieloide crônica, este último também para leucemia linfoblástica aguda Philadelphia positivo, e rituximabe para linfoma não Hodgkin.

Quadro 3. Demandas para Citec/Conitec em oncologia de 2006 a 2013

\begin{tabular}{|c|c|c|c|}
\hline TEMAS AVALIADOS & $\begin{array}{c}\text { CITEC } \\
(2006-2011)\end{array}$ & $\begin{array}{l}\text { CONITEC } \\
(2011-2013)\end{array}$ & TOTAL \\
\hline \multicolumn{4}{|l|}{$\begin{array}{l}\text { Tipos de tecnologia } \\
\text { avaliada }\end{array}$} \\
\hline Procedimento/processo & $6(30 \%)$ & $3(12 \%)$ & $9(17 \%)$ \\
\hline Produto & $2(10 \%)$ & $4(9 \%)$ & $6(11 \%)$ \\
\hline Medicamento & $12(60 \%)$ & 27 (79\%) & $39(72 \%)$ \\
\hline Total & 20 & 34 & 54 \\
\hline \multicolumn{4}{|l|}{ Demandante } \\
\hline Interno (Estado) & $6(30 \%)$ & $8(23,5 \%)$ & $14(26 \%)$ \\
\hline $\begin{array}{l}\text { Externo (Ind. } \\
\text { farmacêutica) }\end{array}$ & $14(70 \%)$ & $26(76,5 \%)$ & $40(74 \%)$ \\
\hline \multicolumn{4}{|l|}{$\begin{array}{l}\text { Aprovações } \\
\text { (incorporação) }\end{array}$} \\
\hline Procedimento/processo & 4 & 1 & 5 \\
\hline Produto & - & 1 & 1 \\
\hline Medicamento* & 5 & 2 & 7 \\
\hline \multicolumn{4}{|l|}{ Tipos de câncer** } \\
\hline Próstata & 2 & 1 & 3 \\
\hline Mama & 1 & 8 & 9 \\
\hline Colorretal & - & 3 & 3 \\
\hline Pulmão & - & 3 & 3 \\
\hline Estômago & 4 & 2 & 6 \\
\hline Leucemia/linfoma & 6 & 4 & 10 \\
\hline Colo Uterino & 3 & 4 & 7 \\
\hline Tireoide & - & 2 & 2 \\
\hline Sistema nervoso central & - & 2 & 2 \\
\hline Pele (melanoma) & - & 1 & 1 \\
\hline Ovário & - & 1 & 1 \\
\hline Cabeça e pescoço & - & 1 & 1 \\
\hline Renal & - & 1 & 1 \\
\hline Fígado & 4 & - & 4 \\
\hline Total & 20 & 33 & 53 \\
\hline
\end{tabular}

* Incorporação de um mesmo medicamento para indicações clínicas ou tipos de cânceres distintos. ** Os tipos de cânceres foram organizados segundo ordem de incidência da doença, tendo o câncer de próstata maior incidência.
A Conitec, de 2011 a 2013, recebeu 34 solicitações, e 33 estavam relacionadas ao tratamento e/ou diagnóstico do câncer. Dessas, 17 foram recusadas por não conformidade dos documentos exigidos, sendo somente oito processos finalizados: três com decisão para não incorporação ao SUS e cinco favoráveis à incorporação. A Conitec, até o final de 2013, havia recomendado somente dois medicamentos para o tratamento do câncer - o alfainterferona, para o tratamento de melanoma cutâneo, e o trastuzumabe, para o tratamento do câncer de mama em fase inicial - para incorporação no SUS. Ressalta-se que em torno de $79 \%$ das demandas para incorporação são de medicamentos. Além disso, não foi gerada nenhuma demanda de solicitação de exclusão de tecnologias em oncologia.

\section{Conclusão}

O Decit/SCTIE/MS é o responsável pela pesquisa em Avaliação de Tecnologias em Saúde (ATS) e de Estudos Econômicos no Brasil. Pode-se inferir que há avanços nesse campo pelo governo brasileiro; outrossim, constatam-se:

- Regulamentação da PNGTS;

- Cooperações internacionais como a oficialização da participação do Ministério da Saúde no International Network of Agencies for Health Technology Assessment (INAHTA);

- Desenvolvimento institucional com a criação das Redes de Pesquisa e Desenvolvimento Nacional;

- Desenvolvimento institucional dos Núcleos de Avaliação de Tecnologias em Saúde (NATS);

- Procis;

- Financiamento de estudos e pesquisas por meio de editais próprios;

- Harmonização e/ou padronização de métodos por meio da elaboração de guias e manuais;

- Definição de parâmetros para priorização de pesquisa em saúde, fundamentada nos critérios de relevância epidemiológica, relevância para a política e para os serviços de saúde, conhecimento avançado sobre o tema e viabilidade operacional;

- Aumento de demandas para incorporação de novas tecnologias fundamentadas oficialmente em AE e ATS.

O fomento à pesquisa na área oncológica é fundamental para a disseminação e o fortalecimento de núcleos de pesquisa independentes e especializados em AE e ATS. Entretanto, alguns tipos de câncer de importância epidemiológica, no contexto nacional, ainda não possuem projetos/estudos de avaliação econômica financiados pelo Decit/SCTIE/MS e/ou demandas de incorporação tecnológica.

A região Sudeste continua a concentrar grande parte dos investimentos em pesquisa de AE e ATS em oncologia, tendo o estado de São Paulo sido contemplado com o maior 
número de projetos. A produção técnico-científica de AE e ATS apresenta um desalinhamento, carecendo ainda de uma agenda com participação social ampla e transparente. Para melhorar os impactos da pesquisa em ATS, são necessárias ações que promovam uma melhor articulação entre a produção científica e os diferentes setores sociais estratégicos.

Por fim, há a necessidade de se aprimorarem as políticas e estratégias relacionadas ao processo de incorporação e de abandono de tecnologias, principalmente de tecnologias em saúde na área oncológica. As políticas científicas de controle do câncer devem estar cada vez mais alinhadas com as evidências científicas com objetivo de oferecer um sistema de saúde equânime e eficiente.

\section{Referências bibliográficas}

Banta HD, Luce BR. Health care technology and its assessment: an international perspective. New York: Oxford University Press; 1993.

Berg M, Grinten T, Klazinga N. Technology assessment, priority setting, and appropriate care in Dutch health care. Int J Technol Assess Health Care. 2004;20(1):35-43.

Brasil. Departamento de Ciência e Tecnologia, Secretaria de Ciência e Tecnologia e Insumos Estratégicos do Ministério da Saúde (DECIT). Avaliação de Tecnologias em Saúde: institucionalização das ações no Ministério da Saúde. Rev Saúde Pública. 2006a;40(4):743-7.

Brasil. Ministério da Ciência, Tecnologia e Inovação. Institutos Nacionais de Ciência e Tecnologia - INCT. Brasília: CNPQ; 2013a. Disponível em: <http://estatico.cnpq.br/programas/inct/_apresentacao/docs/ livro2013.pdf $>$.

Brasil. Ministério da Saúde. Rede Brasileira de Avaliação de Tecnologias de Saúde (REBRATS), 2013b. Disponível em: <http://rebrats.saude.gov.br / quem-somos>. Acessado em: jan. 2013.

Brasil. Ministério da Saúde. Secretaria de Ciência, Tecnologia e Insumos Estratégicos. Departamento de Ciência e Tecnologia. Avaliação de tecnologias em saúde: seleção de estudos apoiados pelo Decit/ Ministério da Saúde, Secretaria de Ciência, Tecnologia e Insumos Estratégicos, Departamento de Ciência e Tecnologia. Brasília: Ministério da Saúde; 2011. 116p. (Série F. Comunicação e Educação em Saúde) ISBN 978-85-334-1809-7.
Brasil. Ministério da Saúde. Secretaria de Ciência, Tecnologia e Insumos Estratégicos. Departamento de Ciência e Tecnologia. Matriz Combinada: um instrumento para definição de prioridades de pesquisa em saúde 1 a ed. Brasília: Ministério da Saúde; 2006b. 87p. (Série D. Reuniões e Conferências).

Brasil. Secretaria de Atenção à Saúde. Instituto Nacional de Câncer. Coordenação de Prevenção e Vigilância. A situação do câncer no Brasil. Rio de Janeiro, 2006c. 119p.

Chinitz D. Health technology assessment in four countries: response from political science. Int J Technol Assess Health Care. 2004;20(1):55-60.

Fleurence RL, Torgerson DJ. Setting priorities for research. Health Policy. 2004;69(1):1-10.

Gadelha CAG. Desenvolvimento, complexo industrial da saúde e política industrial. Rev Saúde Pública. 2006;40(n. esp.):11-23.

Goss PE, Lee BL, Badovinac-Crnjevic T, Strasser-Weippl K, Chavarri-Guerra Y, St Louis J, et al. Planning cancer control in Latin America and the Caribbean. Lancet Oncol. 2013;14(5):391-436.

Guimarães R. Pesquisa em saúde no Brasil: contexto e desafios. Rev Saúde Pública. 2006:40(n. esp.):3-10.

Kaplan W, Laing R. Priority medicines for Europe and the world. 2004.

Montorzi G. Priority Setting for Research for Health: a management process for countries. Geneva: Council on Health Research for Development; 2010.

Novaes HMD, Elias FTS. Uso da avaliação de tecnologias em saúde em processos de análise para incorporação de tecnologias no Sistema Único de Saúde no Ministério da Saúde. Cad Saude Publica. 2013;29 Sup:S7-S16.

Paim J, Travassos C, Almeida C, Bahia L, Macinko J. O sistema de saúde brasileiro: história, avanços e desafios. Lancet. 2010;11-31.

Pichon-Riviere A, Augustovski F, Rubinstein A, Marti SG, Sullivan SD, Drummond MF. Health technology assessment for resource allocation decisions: are key principles relevant for Latin America? Int J Technol Assess Health Care. 2010;26(4):421-7.

Sestelo JAF, Souza LEPF, Bahia L. Saúde suplementar no Brasil: abordagens sobre a articulação público/privada na assistência à saúde. Cad Saude Publica. 2013;29(5):851-66.

Silva HP, Petramale CA, Elias FTS. Avanços e desafios da Política Nacional de Gestão de Tecnologias em Saúde. Rev Saúde Pública. 2012;46:83-90.

Silva LK. Avaliação tecnológica e avaliação de custo-efetividade em saúde: a incorporação de tecnologias e a produção de diretrizes clínicas para o SUS. Ciênc Saúde Coletiva. 2003;8:501-20. 